\title{
Globe
}

Revue internationale d'études québécoises

\section{Homosexualités et reconnaissance sociale : perspectives canadiennes et québécoises}

\section{Joseph J. Lévy et Catherine De Pierrepont}

Volume 12, numéro 2, 2009

Images et représentations de la sexualité au Québec

URI : https://id.erudit.org/iderudit/1000712ar

DOI : https://doi.org/10.7202/1000712ar

Aller au sommaire du numéro

Éditeur(s)

Globe, Revue internationale d'études québécoises

ISSN

1481-5869 (imprimé)

1923-8231 (numérique)

Découvrir la revue

Citer cette note

Lévy, J. J. \& De Pierrepont, C. (2009). Homosexualités et reconnaissance sociale : perspectives canadiennes et québécoises. Globe, 12(2), 159-167.

https://doi.org/10.7202/1000712ar d'utilisation que vous pouvez consulter en ligne.

https://apropos.erudit.org/fr/usagers/politique-dutilisation/ 


\title{
NOTE CRITIQUE
}

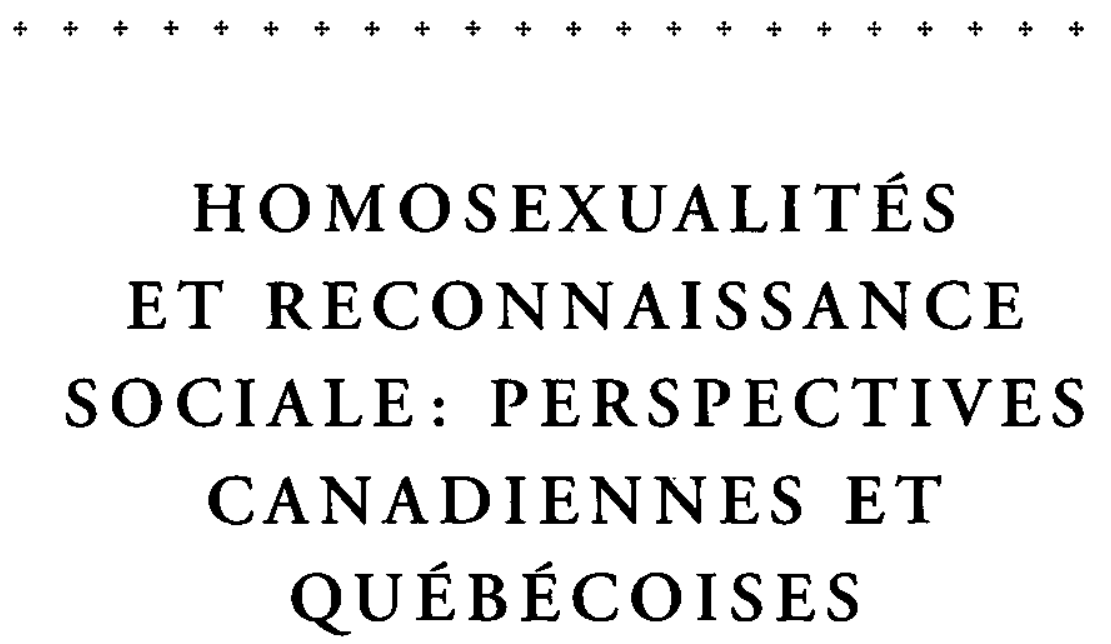

\author{
JOSEPH J. LÉVY \\ Universıté du Québec à Montréal \\ CATHERINE DE PIERREPONT \\ Universıcé du Québec à Montréal
}

$++4++++4++4+++4++4++4++$

Dans les sociétés post-industrialisées, les questions entourant la construction des identités de genre, l'érotisme et la reproduction ont suscité des débats politiques, biomédicaux, juridiques et éthiques où se confrontent des visions de moins en moins consensuelles. Elles s'accompagnent d'une remise en question profonde des idéologies, des modèles et des normes fondées sur le primat de l'hétérosexualité, du mariage et de la reproduction. On assiste aussi à une redéfinition des rapports entre sphères privée et publique, des formes du lien sexuel et amoureux et à des débats sur la pornographie, la prostitution et les violences sexuelles. Pour reprendre le titre de la perspective 
de Michel Foucault dans son histoire de la sexualitél, la volonté de savoir, liée au développement d'une scientia sexualis, n'est plus le fait de chercheurs marginaux, mais elle s'inscrit dans de nombreuses perspectives disciplinaires, depuis l'anthropologie jusqu'aux sciences politiques, et ce, à partir de points de vue théorique et méthodologique diversifiés. Les publications dans ce domaine sont légion, comme le suggèrent la multiplication des ouvrages (dictionnaires, livres, collectifs, monographies, dossiers de revue, articles) qui traitent de thématiques de plus en plus variées, un mouvement auquel le Québec n'échappe pas ${ }^{2}$. Plusieurs publications se sont penchées en particulier sur la question des minorités homosexuelles et nous retiendrons ici trois d'entre elles qui présentent des facettes complémentaires de l'évolution de la question homosexuelle en France, au Québec et au Canada ${ }^{3}$. La première, celle de Patrice Corriveau, La répression des homosexuels au Québec et en France. Du bûcher à la mairie, porte un titre qui met en exergue la perspective théorique et les dimensions comparatives et évolutives privilégiées dans cet ouvrage ${ }^{4}$. À l'origine de sa thèse de doctorat, dont est issu ce livre, un incident homophobe à son encontre, lors d'une sortie entre amis, amène l'auteur, d'orientation hétérosexuelle, à s'interroger sur la répression à l'égard des homosexuels et à tenter d'en cerner les constructions historiques et

$$
+4
$$

1. Michel Foucault, Histoire de la sexualite. Tome I: La volonté de savoir, Paris, Gallimard, 1976. A l'occasion du $30^{c}$ anniversaire de la parution de cet ouvrage, le Bulletin d'histoire politique paru en 2006 (vol. $15, \mathbf{n}^{\circ}$ l) traite des rapports entre politique et sexualité sous plusieurs angles.

2. Dans les publications récentes, on retiendra l'ouvrage collectif Questions de sexualité au Quebec, sous la direction de Joseph J. LÉvy et André Dupras (Montréal, Liber, 2008), qui comprend 67 textes rédigés par de nombreux collaborateurs, chercheurs, étudiants et intervenants, provenant de la majorité des disciplines en sciences sociales. Ce livre paraît à la veille de l'anniversaire des $\mathbf{4 0}$ ans d'existence du département de sexologie de l'Université du Québec à Montréal, soulignant ainsi sa contribution à la compréhension de la sexualité dans le Québec contemporain. Cet ouvrage met en relief la diversité des études québécoises sur la sexualité avec un net parti pris en faveur des approches quantitatives, bien que les méthodologies plus qualitarives tendent à se développer. Il met en évidence des orientations de recherche portant sur les idéologies, les attitudes et les valeurs, alors que les représentations médiatiques (cinéma, télévision, presse, Internet) ec artisciques (théâtre, peinture, sculpture, etc.) de la sexualité au Québec restent, quant à elles, encore peu explorées.

3. Notons aussi la parucion de deux ouvrages collectifs dans la collection "Santé et Société " des Presses de I'Université du Québec. Le premier, Homosexualités et variations régionales (sous la direction de Danielle JULIEN er Joseph J. LEVY, Québec, 2007), analyse les condicions de vie des gais et lesbiennes vivant en milieu rural ou dans les petites villes, où ils se heurcent à des difficulcés nombreuses dans l'expression de leur identicé, à l'isolement, à la nécessité d'une double vie ex à des obstacles dans l'accès à des services sociaux et de santé. Le second, Intersections, cultures, sexualités et genres (sous la direccion de Shari BROTMAN ec Joseph J. LÉVY, Québec, 2008), dégage les enjeux théoriques et méchodologiques qui entourent l'étude des rapports entre les identités socioculturelles et sexuelles er, à partir d'écudes empiriques, mer en relief la diversité des trajectoires des membres des différentes minorités sexuelles. Le vol. $16, \mathrm{n}^{\circ} 3$ du Bulletin d'histoire politique, sous le titre "Homosexualités et politique au Canada et au Québec", analyse quant à lui l'évolution des modes de régulation socio-juridique des homosexualités, les stratégies de résistance ec de lutte et les objectifs politiques des différents mouvements de revendication.

4. Patrice Corriveau, La répression des homosexuels au Québec et en France. Du bûcher à la mairie, Québec, Éditions du Seprentrion, 2006. 
sociopolitiques qui ont eu cours au fil du temps. Après avoir survolé les conceptions de la Grèce antique jusqu'au XVII siècle, il traite de façon détaillée la période qui va de la Grande ordonnance de 1670 jusqu'à la situation contemporaine en France et au Québec. Privilégiant une perspective constructiviste et sociojuridique, l'auteur analyse ainsi les différentes figures que prend l'homosexualité au cours des siècles, montrant comment ces conduites sont culturellement construites et à quelles modalités de régulation et de sanctions elles donnent lieu. Fondée sur les référents religieux bibliques, une première figure, celle du sodomite, se met en place à partir du Moyen Âge et se maintient jusqu'au XVIII ${ }^{\mathrm{e}}$ siècle pour laisser ensuite la place à d'autres figures, celle du pédéraste, de l'inverti, de l'homosexuel et du gai. Elles s'appuient sur des paradigmes idéologiques qui font appel à des argumentations d'abord religieuses et morales qui continueront de sous-tendre, de façon implicite, les discours médicaux et psychiatriques qui vont alimenter l'étiquetage des conduites homoérotiques à partir du XIX ${ }^{e}$ siècle. Ces constructions vont orienter les justifications des codes juridiques, des régulations et des sanctions à l'égard des individus présentant de telles conduites et donner lieu à des formes de stigmatisation et de discrimination qui se maintiennent encore. $\mathrm{Si}$, dans les contextes grec et romain, la conduite homoérotique obéit moins à une régulation juridique que morale, visant à sanctionner les excès et le caractère passif, c'est avec le Moyen Âge que la répression commence à s'institutionnaliser. Se met alors progressivement en place un dispositif visant à punir pour des raisons religieuses les comportements considérés comme sodomites (s'écartant de la nature et des règles ecclésiastiques fondées sur le mariage et la reproduction). Les motivations politiques sont aussi présentes, la figure du sodomite servant de bouc émissaire pour expliquer les catastrophes naturelles ou pour servir d'exutoire aux problèmes politiques, devenant ainsi l'étranger et l'ennemi, ce qui permet d'assurer le pouvoir de l'Église. Pendant la Renaissance, des cycles de tolérance et de condamnation, en fonction de la perception du désordre social qu'elles sont censées créer, s'exercent sur les conduites homoérotiques. Cette périodicité se maintiendra au fil des époques ultérieures, en France et au Québec. Pendant la période allant de la Grande ordonnance de 1670 à la Conquête britannique, le Québec obéit au même régime législatif qu'en France et le système judiciaire tend à ignorer ce type de conduites, à moins de flagrant délit ou d'acte public, de peur qu'elles ne soient imitées. S'il y a une surveillance, des dénonciations, surtout en France, ainsi que des arrestations, les condamnations sont rares, les règlements hors cour, les lettres de cachet ou l'engagement dans l'armée et la promesse de non-récidive prenant le pas sur les procès ou les exécutions 
(bûcher, échafaud, gibet) associées à des conduites violentes ou au meurtre. Les deux régimes vont par la suite diverger. En France, la Révolution provoque une transformation du système législatif avec la mise en place de principes rationnels, égalitaires et laïques qui entraînent la dépénalisation de la sodomie, mais la répression des comportements homoérotiques ne disparaît pas pour autant, car l'impératif de la protection de la jeunesse contre la prédation des pédérastes fait désormais de ceux-ci une cible politique contre laquelle s'exerce une violence accrue. Dans le contexte canadien-français, la situation est différente: la domination de l'Église, qui détient un pouvoir politique et social important, met l'accent sur les fondements de la famille et du mariage, d'où la condamnation de toutes les pratiques qui s'en éloignent. Linfluence du droit criminel anglais va se faire sentir et les relations homoérotiques seront criminalisées, même entre personnes consentantes. Ainsi, en 1841, le Code criminel impose la peine de mort pour ces pratiques. Cette loi sera amendée par la suite et la peine encourue deviendra l'incarcération à vie, même si la jurisprudence reste limitée à quelques cas. Entre la fin du XIXe siècle et les années 1960, plusieurs transformations vont voir le jour dans ces deux contextes. En France, la psychiatrie théorise sur la figure de l'homosexuel associé au perverti qu'il faut soigner et dont l'anormalité constitue une menace au maintien de la nation. S'il y a peu de condamnations, la surveillance et la censure sont maintenues et s'amplifieront pendant le régime de Vichy en 1942 pour entraîner la pénalisation de l'homosexualité, associée à la souillure er à la perversion de la jeunesse dont elle compromettrait ainsi la santé. Dans le contexte canadien, un changement législatif majeur intervient en 1890, lorsque le code criminel introduit les notions d'attentat à la pudeur, de sodomie, mais surtout de grossière indécence, puisque:

Désormais, c'est l'ensemble des comportements homoérotiques commis par des adultes masculins qui sont condamnables en vertu du crime de grossière indécence. Que les accusés soient consentants ou non, ou que l'acte ait été commis en public ou en privé n'y change rien. Les coupables sont passibles d'un emprisonnement de 5 ans avec la possibilité de se voir administrer des coups de fouet (p. 94).

Cette condamnation subsistera jusqu'en 1969. Jusqu'aux années 1960 , les représentations de l'homosexualité provenant des milieux criminologiques, de la psychiatrie et de l'Église tendent à se compléter pour la considérer comme un péché, un crime ou une maladie (l'American Psychological Association la définissait comme un trouble de personnalité sociopa- 
thique), alors que les hypothèses sur son origine se diversifient (de la génétique aux hormones), tout comme les perspectives de traitement. Si les lieux de rencontre et de socialisation homosexuels se multiplient, les conduites restent soumises à des interdits sociaux et médiatiques. Les arrestations et les condamnations s'intensifient, surtout lors des crises socio-économiques, pendant la période duplessiste marquée par l'influence des valeurs religieuses, mais même durant la Révolution tranquille. À partir du début des années 1970, on assiste à un remodelage profond de la question homosexuelle dans les deux pays, avec comme arrière-fond une internationalisation des revendications liées aux mouvements des gais et lesbiennes, dont la visibilité politique et culturelle augmente malgré les campagnes homophobes, surtout à la suite de l'épidémie du VIH/sida, qui contribue à la stigmatisation des homosexuels. Malgré ce revers, on constate une progression dans la reconnaissance des droits des homosexuels. Dans les deux contextes, on assiste à une dépsychiatrisation et à une décriminalisation des conduites homosexuelles et à la mise en place de lois ou à l'ajout d'articles dans les chartes existantes interdisant toute forme de discrimination sur la base de l'orientation sexuelle (en 1977 au Québec, en 1985 en France et en 1995 au Canada). À partir de la fin des années 1990, le débat se déplace vers la question des formes d'union entre personnes de même sexe avec, au Québec, en 2002, la reconnaissance de l'union civile avec les mêmes droits et obligations que les personnes mariées, alors que les tribunaux en Ontario et au Québec invalident la définition traditionnelle du mariage limitée à une relation hétérosexuelle. En 2004, on assiste au premier mariage gai au Québec, alors qu'au fédéral, un projet de loi précise la définition et les conditions du mariage civil, non sans susciter de nombreuses polémiques, la question du mariage gai entraînant des prises de position opposées. En France, en 1999, le Pacte civil de solidarité (PACS) est institué, qui donne aux couples homosexuels les mêmes droits qu'aux couples hétérosexuels. Si l'égalité juridique semble acquise, l'égalité sociale reste à conquérir; les attitudes homophobes, la discrimination et la violence, tout comme le harcèlement policier restent présents, même si l'opinion publique, surtout au Québec, accepte mieux l'homosexualité.

Louvrage de Corriveau, fondé sur une analyse nuancée, permet de saisir les étapes de cette longue marche vers l'égalité en mettant en perspective les différents modes de régulation en fonction des contextes nationaux et la diversité des discours qui ont sous-tendu la stigmatisation des homosexuels. Selon l'auteur, cependant, on assisterait à un déplacement de l'aversion autrefois suscitée par les conduites homoérotiques vers les figures des pédophiles et des pères incestueux, qui deviendraient les nouveaux boucs émissaires dans 
un système social et pénal où la protection de la jeunesse est un impératif. Cette thèse reste cependant à peine ébauchée et une argumentation plus serrée aurait été souhaitable pour l'étayer.

Deux autres ouvrages viennent aider à préciser certains thèmes dégagés par Patrice Corriveau. Le premier, Mariage homosexuel. Les termes $d u$ débat, est un recueil de 45 textes choisis par Guy Ménard ${ }^{5}$, qui ont été soit demandés à des collaborateurs, soit publiés par le journal Le Devoir, soit reçus par la rédaction. On retrouve dans cet ouvrage les différents paradigmes et arguments qui ont été débattus dans l'espace public au sujet du mariage homosexuel. Alors que le paradigme juridique soulève des enjeux touchant l'égalité des citoyens en regard de la loi et de ses limites, le paradigme religieux oppose les tenants du modèle chrétien et catholique fondamentaliste et ceux qui sont partisans d'arrangements doctrinaux dans ce domaine. Le paradigme historique, quant à lui, fait ressortir deux perspectives: d'une part, celle de la pérennité du mariage hétérosexuel et de son immuabilité, d'autre part, celle de la multiplicité de ses formes historiques, à l'encontre d'une approche plus naturaliste et sociobiologique. Le paradigme des sciences humaines privilégie l'analyse des conditions sociologiques et sociopsychologiques pour situer les enjeux touchant la remise en question du modèle familial dominant, reflétant soit "l'individualisme narcissique" comme mode de revendication, soit au contraire la diversité des formes de relations possibles dans les sociétés modernes où l'idéologie des droits individuels s'arrime à la souveraineté du sujet dans ses choix de vie. Les débats font aussi rage dans les milieux homosexuels où la question du mariage ne fait pas l'unanimité et oppose ceux qui désirent maintenir l'exceptionnalité de la condition homosexuelle et ceux qui voient dans cette revendication un moyen d'assurer l'égalité des droits, de diversifier les styles de vie et de signifier l'égalité sociale citoyenne. L'ensemble de ces riches débats met bien en relief la diversité des constructions socioculturelles de l'homosexualité et les stratégies argumentaires utilisées pour fonder les points de vue.

Le livre Mariage gai. Les coulisses d'une révolution sociale ${ }^{6}$, de Sylvain Larocque, approfondit notre compréhension des dimensions sociopolitiques de la question, et ce, à partir d'une perspective journalistique. S'appuyant sur une trentaine d'entrevues et de témoignages avec des personnes clés impliquées dans ce dossier à différents niveaux, de la base aux décideurs politiques (ministres et conseillers politiques), Larocque décrit

$$
+4
$$

5. Guy MÉNARD, Mariage homosexuel. Les termes du debat, Montréal, Liber, 2003.

6. Sylvain LAROCQUE, Mariage gai. Les coulisses d'une révolution sociale, Paris, Flammarion, 2006. 
rétrospectivement les luttes et les débats qui ont mené à la reconnaissance légale du mariage gai, qui ouvre la voie à une plus grande acceptation sociale de l'homosexualité. Au fil des chapitres, l'auteur dépeint l'arrière-fond socioculturel, marqué par les différentes formes d'homophobie, dans lequel évoluent les acteurs du débat, complétant ainsi le tableau dressé par Patrice Corriveau. Il décrit les principales étapes historiques, les discussions et les stratégies qui ont abouti à l'acceptation légale du mariage gai dans le contexte canadien. Le livre montre ainsi comment les actions entreprises par les différentes instances, des juges aux simples citoyens en passant par les leaders des mouvements des gais et des lesbiennes, ont contribué, à long terme, aux modifications qui ont mené à la situation actuelle, dans un contexte sociopolitique où les victoires et les défaites dans le processus d'accession à l'égalité juridique se succédaient, ponctuant un parcours marqué par les tensions entre l'opinion publique, les groupes de pression en faveur ou en défaveur du mariage homosexuel, les instances juridiques, tant fédérales que locales, et les décideurs politiques, ces derniers étant, pour un grand nombre d'entre eux, plus conservateurs que le reste de la population. Louvrage met aussi au jour la complexité des stratégies qui parcourent cet itinéraire: avis des experts, décisions des juges, mémoires de différents groupes, actions ponctuelles de citoyens, délibérations parlementaires et votes. Ces interventions juridiques, politiques et citoyennes ont constitué l'arsenal des moyens auxquels ont eu recours les différents acteurs pour faire valoir leurs points de vue. Elles mettent en évidence toutes la panoplie des armes disponibles, dans le contexte démocratique, pour faire valoir une cause ou s'y opposer. Les différents camps se sont inspirés d'études historiques, anthropologiques, religieuses ou morales pour développer leurs arguments. Des juges, arbitres incontournables, ont aidé, avec leurs décisions successives, à défaire les arguments essentialistes et naturalistes qui faisaient du mariage hétérosexuel et de la procréation le fondement de la famille - une perspective discriminatoire inacceptable dans une société libre et démocratique - et ont ouvert la voie, en 2002, à l'union civile entre homosexuels. Ce jugement a été porté en appel par le gouvernement canadien, qui a alors été accusé de lâcheté et d'évitement face à ses responsabilités. Le débat s'est ensuite déplacé surtout au plan politique et, si les députés du caucus au pouvoir étaient ambivalents, de plus en plus de ministres ont appuyé la position en faveur du mariage homosexuel. L'évolution de cette tendance a abouti en 2002 à la mise en place d'un comité parlementaire en vue d'une consultation publique sur cette question, où ont témoigné et pris position divers groupes d'acteurs sociaux (près de 500 témoins dans dix villes). Les opposants conservateurs, 
dont les arguments reprenaient des antiennes moralistes et religieuses, ont souligné les répercussions négatives du mariage gai sur la société et la famille, tandis que ses partisans ont fait valoir des contre-arguments plus progressistes. À la même époque, la Colombie-Britannique, rejoignant en cela le Québec et l'Ontario, s'est prononcée en faveur de ce type de mariage tout en imposant un moratoire de deux ans, tandis que la cour d'appel de l'Ontario accordait aux couples homosexuels la possibilité de se marier sous décision judiciaire. Cette décision a renforcé la position des tenants de la reconnaissance juridique que préconisait aussi le comité parlementaire qui considérait que «le temps [était] venu de changer la définition du mariage afin de permettre le mariage entre conjoints de même sexe" (p. 133), sans que cette perspective ne fasse consensus, les oppositions étant encore vives. Jean Chrétien, premier ministre du Canada de l'époque, fortement opposé à l'idée du dépôt d'une loi sur le mariage gai, malgré l'appui majoritaire de ses ministres, en particulier celui du ministre de la Justice Martin Cauchon, a continué de tergiverser, avant de faire une annonce officielle le 17 juin 2003:

Nous n'appellerons pas de la récente décision sur la définition du mariage [...]. Au lieu de cela, nous allons proposer une loi qui protégera les droits des Églises et des organisations religieuses de sanctifier le mariage tel qu'elles le définissent. En même temps, nous allons veiller à ce que notre loi inclue et reconnaisse en droit l'union des couples homosexuels. Dès que le projer de loi aura été rédigé, il sera renvoyé à la Cour suprême, après quoi il sera soumis à un vote libre à la Chambre (p. 167-168).

Cette décision a été accueillie comme un événement historique par plusieurs alors que d'autres ont continué de s'y objecter. Le projet de loi a été transmis à la Cour Suprême du Canada pour qu'elle valide la démarche et réponde à trois questions:

1. La définition du mariage relève-t-elle de la compétence exclusive du Parlement fédéral?

2. Le mariage homosexuel est-il conforme à la Charte des droits et libertés?

3. La liberté de religion, inscrite dans la Charte, permet-elle aux autorités religieuses de refuser le mariage de conjoints du même sexe? (p. 175)

La loi a ensuite été soumise au Parlement, tandis que partisans et opposants s'organisaient dans l'espace public, reflétant les antagonismes présents à la 
Chambre des communes. Le premier vote, accompagné de débats houleux, n'a pu départager les dépurés, le nombre de vores pour et contre la motion ayant été égal, mais au second tour, les oppposants au projet ont été plus nombreux. À la fin de l'année 2003, un nouveau gouvernement, avec Paul Martin comme premier ministre, a été formé et de nouvelles péripéties judiciaires sont survenues, tant au fédéral qu'au provincial. Plusieurs territoires et provinces, comme le Yukon et la Saskatchewan, ont permis le mariage gai. Ils ont été imités par la Nouvelle-Écosse et Terre-Neuve puis, plus tardivement, par le Nouveau-Brunswick. La Cour Suprême a répondu par l'affirmative aux questions qui lui avaient été posées, sauf à celle qui visait à déterminer si la question du mariage traditionnel viole la Charte des droits et libertés. Ainsi, malgré les résistances de l'opposition et d'une partie du public qui refusait cette légalisation, le projet de loi sur le mariage civil (C-38) a été présenté à la Chambre des communes en février 2005. Il réitérait l'idée que " [l]e mariage est, sur le plan civil, l'union légitime de deux personnes, à l'exclusion de toute autre [et] que les autorités religieuses sont libres de refuser de procéder à des mariages non conformes à leurs convictions» (p. 264-265). Après d'autres débats et le rejet d'un amendement, il a été adopté par une majorité de députés et accueilli comme une avancée sociale importante, puis il a été resoumis en troisième lecture et approuvé avant d'être présenté au Sénat, qui a ratifié la Loi, avant qu'elle reçoive la sanction royale et entre ainsi en vigueur.

Cette égalité juridique, qui demande à être confirmée au plan social, met en relief la complexité des positions et des arguments de chaque camp, fournissant ainsi une illustration des processus en jeu dans l'espace public contemporain, où une diversité de groupes d'acteurs interviennent pour orienter et canaliser les débats, contribuant ainsi au développement d'une "démocratie sexuelle" où le "sens social s'invente sous nos yeux, à mesure que se déploient les batailles qui composent notre actualité, sans jamais dessiner une image stable, consensuelle et définitive: c'est la logique politique sans fin de la démocratisation sexuelle ${ }^{7}$. Cette démocratisation sexuelle, qui fait appel à la fois aux discours et aux passions, constitue un révélateur remarquable des enjeux de citoyenneté dans les pays postindustrialisés, ouvrant ainsi la voie aux revendications d'autres minorités sexuelles encore mal connues sur le plan sociohistorique.

$+++$

7. Clarisse FABRE et Éric FASSIN, Liberté, egalité, sexualités. Actualités politiques des questions sexuelles, Paris, Belfond, 2003, p. 230. 